\title{
Probing The Mechanical Properties of Few-Layer Graphene with Aberration- Corrected, Low-Voltage STEM
}

\author{
Edmund Han ${ }^{1}$, Jaehyung Yu², Kayla Nguyen ${ }^{1}$, Elif Ertekin ${ }^{2}$, Arend van der Zande ${ }^{2}$ and Pinshane Y. \\ Huang $^{1 *}$
}

1. Department of Materials Science and Engineering, University of Illinois Urbana-Champaign, Urbana, IL, USA.

2. Department of Mechanical Science and Engineering, University of Illinois Urbana-Champaign, Urbana, IL, USA.

* Corresponding author: pyhuang@illinois.edu

Understanding the deformability, flexibility, and bending mechanics of two-dimensional (2D) materials is critical for the realization of next-generation deformable electronics and nanomechanical devices. While the mechanics of few-layer graphene have been studied for more than a decade, there is still no consensus on its bending stiffness and how it scales with thickness [1-3]. Conventional measurements from mechanical resonance and nanoindentation [4] are challenging because out-of-plane deformations and pre-tension strongly impact the extraction of bending stiffness. Electron microscopy provides a powerful platform for addressing this challenge by enabling measurements of the conformation and strain of 2D materials at atomic resolution. Using aberration-corrected STEM at $80 \mathrm{kV}$, below the knock-on damage threshold of graphene [5], we investigate the bending mechanics of few-layer graphene. Using low voltages and dose rates, we probe the graphene on the atomic scale while minimizing electron beam damage in order to measure the equilibrium conformation of highly curved 2D materials. Using a combination of STEM, scanning convergent beam diffraction, geometric phase analysis, and density functional theory (DFT) we show that the bending of few-layer graphene is dominated by slip and shear rather than in-plane strain. As a result, few-layer graphene exhibits unusual, curvature-dependent mechanics that can dramatically tune its bending stiffness; for example, the stiffness of trilayer graphene changes by almost $300 \%$ when it is curved from 6 to 50 degrees.

We explore how bending stiffness of few-layer graphene varies with thickness and bending angle by systematically examining its conformation over atomically-sharp hexagonal boron nitride (h-BN) steps, as shown in Figures 1a-b. These geometries offer direct control over two key variables: number of graphene layers and h-BN step height. Using aberration-corrected STEM, we measure the bending profiles of few-layer graphene (Figures 1c-h) and extract geometric parameters including the bending angles and radii of curvature. We use these values to calculate the bending stiffness as a function of thickness (Figure 2a).

Our experimental data and DFT calculations reveal surprising bending phenomena in few-layer graphene. First, rather than being a single value at a given thickness, the bending stiffness decreases as a function of the curvature angle (Figure $2 b-c)$. Second, we observe a change in thickness-dependent scaling of the graphene bending stiffness from quadratic (below $6^{\circ}$ ) to linear (above $42^{\circ}$ ). We attribute this transition to a decrease in the interlayer coupling as the material is curved and the atomic registry between adjacent layers is reduced. These properties result directly from shear and slip between the individual atomic planes of graphene, where the energetic barrier for slip is more than an order of magnitude lower than in metals. Our results indicate that the bending stiffness of few layer graphene can be orders of magnitude smaller 
than previously thought and provide a new lower limit for the fabrication of ultra-soft, high mobility electronic nanodevices based on 2D materials [6].

\section{References:}

[1] D Akinwande et al., Extreme Mechanics Letters 13 (2017), p. 42.

[2] X Chen et al., Appl. Phys. Lett. 106 (2015), p. 101907.

[3] D-B Zhang et al., Phys. Rev. Lett. 106 (2011), p. 255503.

[4] M Poot and HSJ van der Zant, Appl. Phys. Lett. 92 (2008), p. 063111.

[5] JC Meyer et al., Phys. Rev. Lett. 108 (2013), p. 239902.

[6] This work was supported by the NSF-MRSEC program under NSF Award Number DMR-1720633. Experiments were carried out in the Frederick Seitz Materials Research Laboratory at the University of Illinois at Urbana-Champaign.
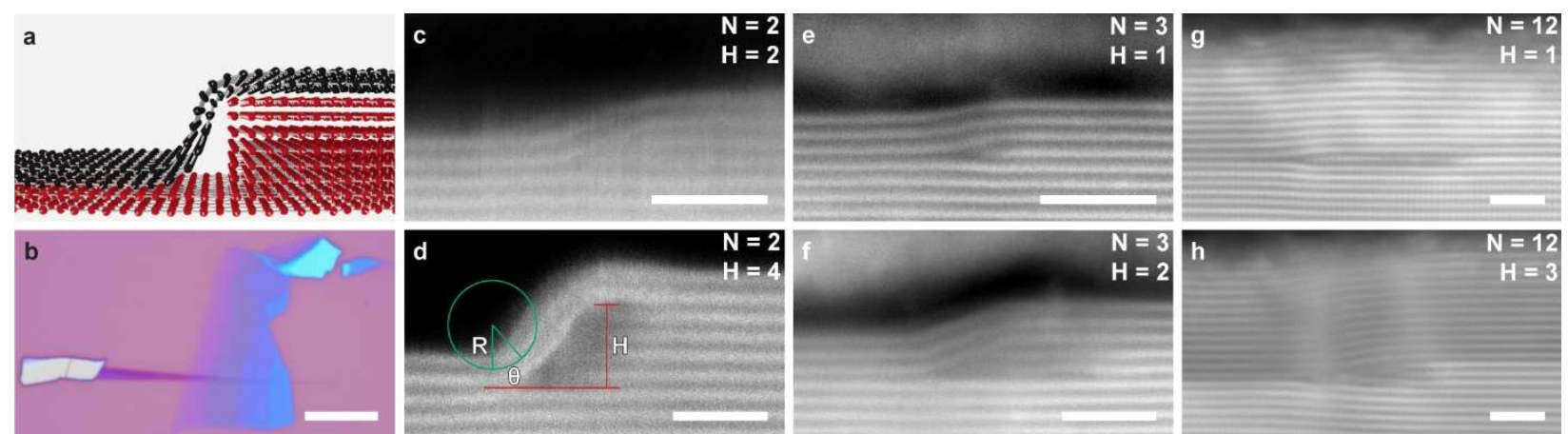

Figure 1. Graphene-hBN heterostructure to experimentally measure bending stiffness. a) Schematic of bilayer graphene draped over an atomically-sharp h-BN step. b) Optical image of graphene transferred onto h-BN flake. c-h) ADF-STEM images of $N$-layer graphene on h-BN step that is $H$ layers tall. Radius of curvature $\mathrm{R}$, bending angle $\theta$, and step height $\mathrm{H}$ are measured as indicated in Figure 1d and used to extract bending stiffness.
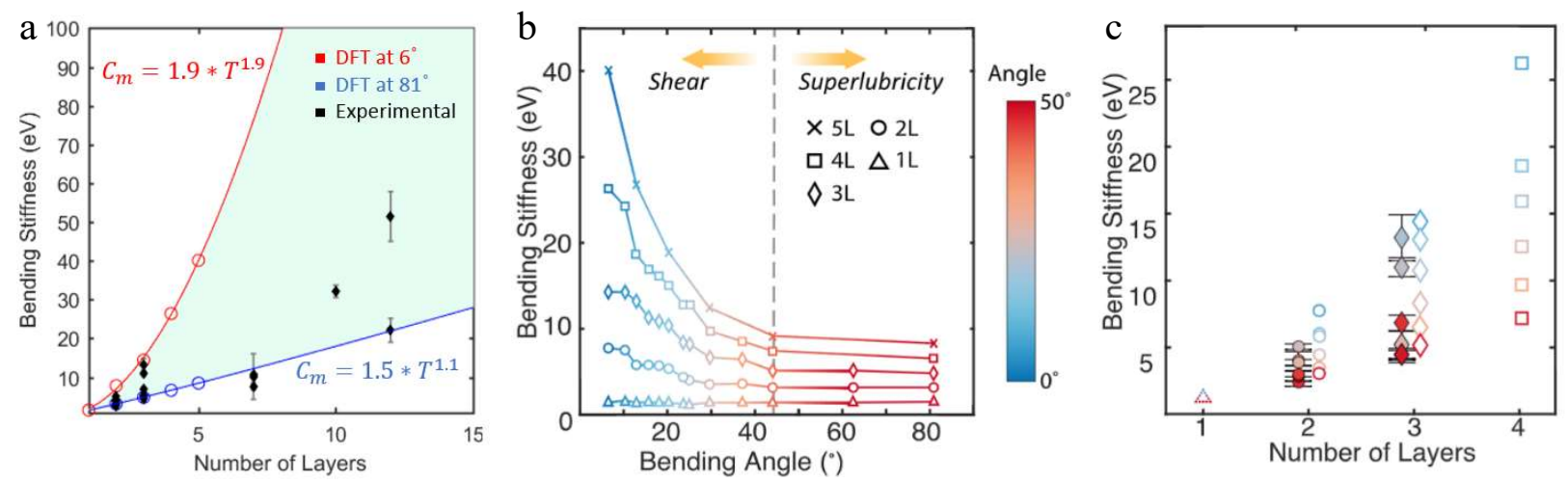

Figure 2. Experimental and DFT calculations of bending stiffness of few-layer graphene. a) Bending stiffness vs. FLG thickness. Error bars indicate 95\% confidence interval. b) DFT-calculated bending stiffness vs. bending angle for monolayer to five-layer graphene, showing dramatic softening with increased angle of curvature. c) Comparison of experimental (solid) and DFT (hollow) bending stiffness vs. thickness for monolayer to four-layer graphene, color-coded with bending angle. DFT and experimental results show qualitative and quantitative agreement in how bending stiffness varies with thickness and bending angle. 